SNUTP-02/036

\title{
Softening of SUSY flavor problem from asymptotically free bulk interactions
}

\author{
Kang-Sin Choi* Ki-Young Choi ${ }^{\dagger}$ and Jihn E. Kim ${ }^{\ddagger}$ \\ Physikalisches Institut, Universität Bonn, \\ Nussallee 12, D53115, Bonn, Germany, and \\ School of Physics and Center for Theoretical Physics, \\ Seoul National University, Seoul 151-747, Korea
}

(March 2, 2019)

\begin{abstract}
Recently, it was pointed out that soft masses of the supersymmetric gauge theories with extra dimensions tends to a flavor conserving point, which is a desirable scenario in gravity mediation models. We point out that in $6 \mathrm{D}$ we must consider the anomaly free condition in addition to the condition on the asymptotic freedom. From this, we find $E_{6}, E_{7}$ and $E_{8}$ are natural candidates in $6 \mathrm{D}$. There is no $S U(N)$ model, but there exist two $S O(10)$ models and $S O(2 n)$ models(one each for each $n \geq 6$ ) satisfying these conditions. In 5 dimensions, there is no such condition on anomaly freedom, but the softening may not be enough.
\end{abstract}

\footnotetext{
*ugha@th.physik.uni-bonn.de

${ }^{\dagger}$ ckysky@th.physik.uni-bonn.de

†jekim@phyp.snu.ac.kr, jekim@th.physik.uni-bonn.de
} 
[Key words: SUSY flavor problem, extra dimensions, 6D gauge anomaly]

PACS: 04.50.+h, 12.20.-g, 11.30.Hv, 12.60.Jv

Typeset using REVTEX 


\section{INTRODUCTION}

The flavor problem in the gravity mediation scenario has to be resolved if it is going to descibe the soft terms of the minimal supersymmetric standard model(MSSM) successfully. In the 4 dimensional (4D) supergravity models, this has been known to be an extremely difficult problem [1]. With the advent of new tries on extra dimensions [2], this flavor problem can be reconsidered toward a possible understanding of the SUSY flavor problem.

The recent 'extra dimensional scenarios' are based on the hope that these extra dimensional field theories are obtainable from compactifications of 10D superstring models or 11D M-theory [3]. In the early string models, it was argued [4] that the GUT scale $M_{G U T}$, the string scale $M_{s}$, and the reduced Planck scale $M_{P}$ are considered to be of the same order, under the assumption that a 4D SUSY field theory is obtained from a 10D SUSY field theory which in turn is considered to be a valid effective theory below the string scale $M_{s}$. However, the string scale of order $6 \times 10^{17} \mathrm{GeV}[4]$ is known to be somewhat larger than the unification scale $M_{G U T} \sim 2 \times 10^{16} \mathrm{GeV}$ determined from the renormalization group running of the observed low energy couplings.

Contrary to this early prediction on $\mathrm{O}(1)$ number for the mass ratios, phenomenologically we need to introduce a small parameter,

$$
\frac{M_{G U T}}{M_{P}} \simeq 10^{-2}
$$

where $M_{P} \simeq 2.44 \times 10^{18} \mathrm{GeV}$ is the reduced Planck mass. Initially, this small number has been considered to be a problem in perturbative string models. Therefore, Horava and Witten proposed a relatively large $11^{\text {th }}$ dimension with two 9-branes with an $E_{8}$ group at each brane to interpret this small number [5].

Recently, Friedmann and Witten [6] estimated $M_{G U T}$ from the top-down approach with the $11 \mathrm{D}$ supergravity compactified with a $G_{2}$ holonomy. In this top-down calculation, they seem to obtain a small number if $\alpha_{G U T} \simeq 1 / 25$,

$$
\left(\frac{M_{G U T}}{M_{P}}\right) \simeq \alpha_{G U T}^{3 / 2} \times \frac{L(Q)^{1 / 3}}{\sqrt{4 \pi a}}
$$


where $a$ is an appropriate ratio of the $7 \mathrm{D}$ compact internal space and $7 / 3$ power of the 3D internal space of 7D supergravity, and $L(Q)$ is the $\mathrm{O}(1-10)$ number of the lens space. Numerically, then Eq. (2) turns up a number of $\mathrm{O}\left(10^{-2}\right)$. Even though one can argue that the Friedmann-Witten calculation (2) is for a specfic model, it may have some truth in it if the volume of the extra dimension is relatively large. One notable difference of this calculation from that of Ref. [4] is that a 10D SUSY field theory is not considered as an intermediate effective theory. The recent tries of the extra dimensional field theories also do not assume a 10D SUSY field theory.

In Ref. [4], it was pointed out in addition that for a 6D SUSY field theory between the string scale and the GUT scale $(\simeq$ compactification scale $)$, one has $M_{s} / M_{G U T}<O\left(1 / \sqrt{\alpha_{g u t}}\right)$. If $\alpha_{\text {gut }} \sim 1 / 25$, then the scale $M_{G U T}$ can be at most $0.1 M_{s}$, which was the reason that Ref. [4] assumed that even in 6D a small number (1) is unreasonable. However, with a power-law asymptotic freedom above the scale $M_{G U T}, \alpha_{g u t}$ can be much smaller than $\frac{1}{25}$ and a large discrepancy between $M_{G U T}$ and $M_{s}$ can be generated. This power law running was not used in Ref. [4].

With a large internal space volume, many Kaluza-Klein(KK) modes in the bulk can contribute significantly in the running of the gauge couplings, leading to a power law instead of a logarithmic running [7]. If an effective $4 \mathrm{D} \beta$ function contributed by the bulk fields is negative, the corresponding gauge coupling constant decreases very rapidly at shorter distance scales. This can be translated to a ratio between the compactification volume and an appropriate Planck scale. Thus, if the volume of the internal space is large compared to a Planckian volume, there is a chance to understand the small number (1). If the SUSY flavor problem is related to this small number, there is a hope to understand it with extra dimensions.

Indeed, Kubo and Terao [8] investigated the possibility of solving the SUSY flavor problem using the small number (1) without introducing bulk matter. In this paper, we confirm their conclusion even with bulk matter, and obtain several candidate GUT groups in 6D from the conditions on the asymptotic freedom and anomaly freedom. 


\section{THE KUBO-TERAO MECHANISM}

Let us briefly discuss the Kubo and Terao(KT) idea [8] in higher dimensional SUSY field theory models, notably in 6D models. Here, gauge multiplets are put in the bulk and matter multiplets are put only at the branes. To realize this kind of setting from string theory, the compactification creates matter only at the branes. ${ }^{1}$ However, we argue that it is not an absolute requirement to put matter only at the branes. An asymmetric assignment of matter in the bulk and branes can be more flexible in understanding top-bottom mass hierarchy [10], and still a kind of KT mechanism can work, since the essence of the KT mechanism is the asymptotic freedom of the gauge couplings in the bulk and the existence of KK towers from the bulk fields. Note that the threshold effect of Ref. [6] relies only on the topology of the internal space, not needing a knowledge on the KK spectrum, which made it easy to write the answer in the simple form given in $(2){ }^{2}$

The orbifold compactifications toward 4D and 6D models are extensively tabulated in the literature [11]. Two explicit 6D models $\left(S O(16)\right.$ and $\left.E_{7}\right)$ are obtained by a $Z_{2}$ orbifold compactification [3]. Here we study SUSY field theories in 5 or 6 dimensions, but with a keen eye on possible compactifications from 10D string theory or 11D M-theory.

In this paper, we assume that below the string scale $M_{s}$ particle interactions are effectively described by a $(4+\delta)$-dimensional field theory with $\delta$ a small number. Specifically, we will choose $\delta=2$. $M_{s}$ may or may not coincide with the Planck mass $M_{P}=2.44 \times 10^{18}$ $\mathrm{GeV}$, but it is known to be close to $M_{P}[4]$ and we take this viewpoint. We also assume for simplicity that the grand unification scale $M_{G U T}$ is the KK scale $\equiv \frac{1}{R}$ where $R$ is the

\footnotetext{
${ }^{1}$ Indeed, there exists an example close to this requirement in an orbifold compactification [9].

${ }^{2}$ At present, it is not known how to apply the $\mathrm{KT}$ mechanism in the $G_{2}$ holonomy case since a detail knowledge on the KK spectrum in the bulk is not needed in this case.
} 
compactification radius in the sense that the compact volume is $X_{\delta} R^{\delta}$ with $^{3}$

$$
X_{\delta}=\frac{\pi^{\delta / 2}}{\Gamma(1+\delta / 2)} \rightarrow\left(2, \pi, \frac{4 \pi}{3}\right) \text { for } \delta=1,2,3
$$

Namely, the scales have a hierarchy

$$
\begin{aligned}
& \text { (string) } \underset{M_{s}}{\longrightarrow}(\mathrm{d}=4+\delta, \mathrm{N}=1 \text { supergravity }) \\
& \underset{M_{G U T}}{\longrightarrow}(\mathrm{d}=4, \mathrm{~N}=1 \text { supergravity MSSM })
\end{aligned}
$$

Thus, toward a 4D observer at low energy there arise towers of KK states above the scale $M_{G U T}$. Including these states in the running of gauge couplings between $M_{G U T}$ and $M_{s}$, we obtain $[7]$

$$
\alpha_{a}^{-1}(\Lambda)=\alpha_{a}^{-1}\left(\mu_{0}\right)-\frac{b_{a}-\tilde{b}_{a}}{2 \pi} \ln \frac{\Lambda}{\mu_{0}}-\frac{\tilde{b}_{a} X_{\delta}}{2 \pi \delta}\left[\left(\frac{\Lambda}{\mu_{0}}\right)^{\delta}-1\right]
$$

where $b_{a}$ is the beta function coefficient of the group $G_{a}$ contributed by all the MSSM fields, $\tilde{b}_{a}$ is the one contributed by the bulk fields. Keeping the power law divergent term only in asymptotically free models, we obtain

$$
\frac{M_{G U T}}{M_{s}} \longrightarrow \frac{\mu_{0}}{\Lambda} \simeq\left(\frac{-\tilde{b} X_{\delta} \alpha_{a}(\Lambda)}{2 \pi \delta}\right)^{1 / \delta}
$$

The KK sum is from the lowest one $1 / R$ to the highest one $M_{s}$. Thus, the length scale describing the internal space is $R \sim 1 / M_{G U T}$ and the string scale is $1 / M_{s}$, giving a ratio of the compactification volumes as

$$
\frac{M_{G U T}}{M_{s}} \sim\left(\frac{L_{s}}{R}\right)
$$

which is another way of saying that the small number (1) needs a large volume in the extra $\delta$-dimension. For the Friedmann-Witten case (2), we do not obtain this relation exactly even though a large volume effect must be there.

\footnotetext{
${ }^{3}$ Indeed, if a GUT group is broken geometrically as in Ref. [2], $M_{G U T}$ is the scale of the first KK mass.
} 
The gaugino masses evolve as

$$
M_{a}\left(M_{G U T}\right)=\left(\frac{g_{a}\left(M_{G U T}\right)}{g_{a}\left(M_{s}\right)}\right)^{2} M_{a}\left(M_{s}\right) \underset{\tilde{b}<0}{\longrightarrow} \frac{M_{a}\left(M_{G U T}\right)}{M_{a}\left(M_{s}\right)} \simeq \frac{C_{2}\left(G_{a}\right) X_{\delta} \alpha_{G U T}}{\pi \delta}\left(\frac{M_{s}}{M_{G U T}}\right)^{\delta},
$$

and the soft scalar masses evolve as ${ }^{4}$

$$
\left(m^{2}\right)_{j}^{i}\left(M_{G U T}\right)=\frac{C_{2}\left(R_{i}\right)}{C_{2}\left(G_{a}\right)}\left[1-\left(\frac{g_{a}\left(M_{s}\right)}{g_{a}\left(M_{G U T}\right)}\right)^{4}\right] \delta_{j}^{i}\left|M_{a}\left(M_{G U T}\right)\right|^{2}+\left(m^{2}\right)_{j}^{i}\left(M_{s}\right)
$$

where $C_{2}$ is the quadratic Casimir operator, e.g. $C_{2}\left(S U_{5}\right)=5, C_{2}(\mathbf{5})=\frac{12}{5}$ and $C_{2}(\mathbf{1 0})=$ $\frac{18}{5}$. From the gaugino mass evolution (8), we note that due to the small number (1) $M_{a}\left(M_{G U T}\right) / M_{a}\left(M_{s}\right)$ is large if the group $G_{a}$ is asymptotically free. Then, if $\left(m^{2}\right)_{j}^{i}$ is small or comparable to the gaugino mass squared at $M_{s}$, the soft mass term at the scale $M_{G U T}$ is dominated by the diagonal element due to Eq. (9). This relative enhancement of the soft mass from the KK mode contribution in the bulk is the KT scenario of suppressing the flavor changing neutral current. For this scenario to work, one needs an asymptotically free gauge interaction in the bulk and a large internal space volume. In this case, it was also pointed out $[8]$ that the soft $A$ and $B$ terms tend to the flavor conserving points. We confirm that these conclusions are true.

As a numerical guide, we present the evolution of the gaugino mass in 5D in Fig. 1 with all Yukawa couplings set at zero except that of the top quark. We chose a figure with 1.5 $\mathrm{TeV}$ gluino mass at $M_{Z}$. Here, we assumed the MSSM spectrum between $M_{Z}$ and $M_{G U T}$. We use the gluino mass at $M_{Z}$ as an input. For the observed values of the strong, weak and electromagnetic coupling constants, we run the parameters up to $M_{G U T}$. The wino and zino(or photino) masses are obtained by running the unified gaugino mass(the gluino mass) from $M_{G U T}$ down to $M_{Z}$. Between $M_{G U T}$ and $M_{s}$, a SUSY $S U(5)$ is assumed with three families and two Higgs quintets but without a $\mathbf{2 4}_{H}$. [A geometrical breaking of $S U(5)$ does

\footnotetext{
${ }^{4}$ It is required that the bulk matter Yukawa couplings do not dominate over the bulk gauge running. Without bulk matter [8], this condition is satisfied. In the next section, we show that even bulk matter with top quark coupling is not harmful.
} 
not need an adjoint Higgs field.] Indeed, $M_{5}\left(M_{G U T}\right) / M_{5}\left(M_{P}\right)$ is a big number and softening of the flavor changing neutral current is achieved. If we had worked in 6D as in Ref. [8], it would not be a consistent calculation since it has a 6D gauge anomaly. 


\section{FIGURES}

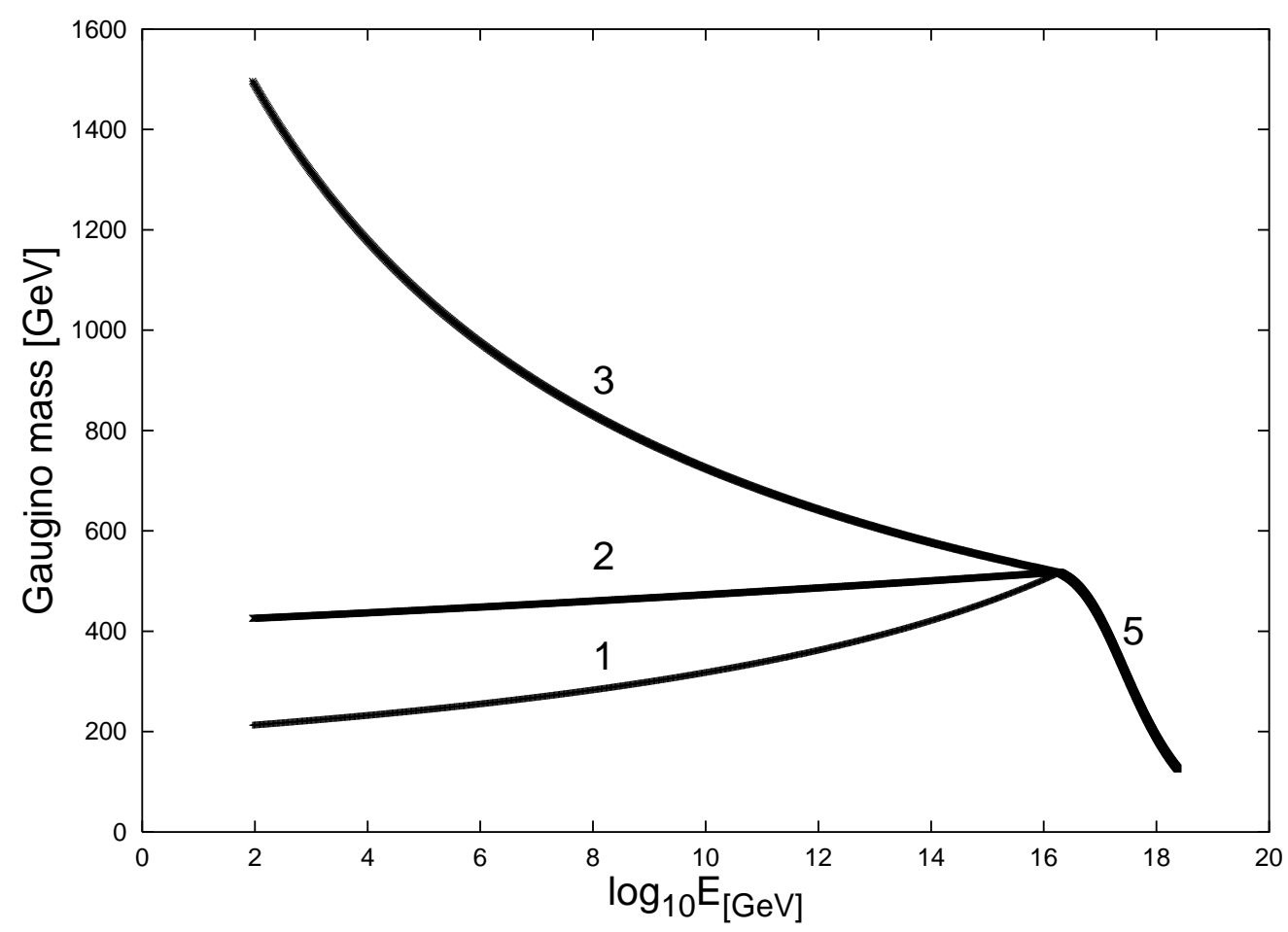

FIG. 1. The evolution of gaugino masses with a 5D $S U(5)$ GUT. $S U(N)$ and $U(1)$ gauginos are marked by $N$ and 1 , respectively.

\section{BULK MATTER CONTRIBUTION}

For the KT scenario to work, the Yukawa couplings should not behave in the same way as the soft mass behavior discussed in Sec. II. Namely, the Yukawa couplings should not be diagonalized so that a reasonable quark mixing matrix is obtained. If there is no bulk matter [8], this differentiation is easily achieved. The main reason for this differentiation is that the soft masses are renormalized additively but the main contribution to the Yukawa coupling renormalization is multiplicative. It is ironic to observe that the very nature of the additive renormalization of scalar masses needed supersymmetry for the gauge hierarchy solution, but the SUSY flavor problem created by supersymmetrization employs this additive renormalization property toward a solution of the SUSY flavor problem. 
On the other hand, if we introduce bulk matter, the discussion is more involved, which we show below. For the bulk matter, we have the following renormalization group equation for the Yukawa coupling $Y^{i j k}[12]$

$$
\begin{aligned}
\Lambda \frac{d Y^{i j k}}{d \Lambda}= & \frac{1}{32 \pi^{2}}\left(Y^{i j l} Y^{k m n}+(j \leftrightarrow k)+(i \leftrightarrow k)\right) Y_{l m n} \\
& +\frac{1}{32 \pi^{2}} \sum_{\{l, m, n\}}\left[X_{\delta}\left(\frac{\Lambda}{M_{G U T}}\right)^{\delta}-1\right]\left(Y^{i j l} Y^{k m n}+(j \leftrightarrow k)+(i \leftrightarrow k)\right) Y_{l m n} \\
& -\frac{2}{16 \pi^{2}}\left(C_{2}\left(R_{i}\right)+C_{2}\left(R_{j}\right)+C_{2}\left(R_{k}\right)\right) G_{\delta}^{2} Y^{i j k}
\end{aligned}
$$

where the dummy index sum is for bulk matter $\{l, m, n\}$, and

$$
G_{\delta}^{2}=g^{2} X_{\delta}\left(\frac{\Lambda}{M_{G U T}}\right)^{\delta}
$$

which becomes constant due to the asymptotically free gauge coupling. The first term of Eq. (10) comes from the zero modes(brane and bulk) of the fields in the loop, the second term comes from the diagrams which contain KK modes in the loop. The third term of Eq. (10) comes from the diagrams which contain gauge fields in the loop(the zero mode and KK modes). Since the first term is logarithmic it can be ignored compared to the other two terms. The second and third terms are the power-running and have different signs. According to the relative magnitudes, the Yukawa coupling can increase or decrease as $\Lambda$ increases. Thus, let us compare the magnitudes of these two terms as the energy scale $\Lambda$ increases from $M_{G U T}$ to $M_{s}$.

These two terms have the following relative magnitudes,

$$
\begin{aligned}
& 28\left[X_{\delta}\left(\frac{\Lambda}{M_{G U T}}\right)^{\delta}-1\right] Y^{2} \\
& -12 C_{2}(r) G_{\delta}^{2} .
\end{aligned}
$$

The coefficient 28 in (12) is obtained for $E_{6}$ Yukawa coupling $\mathbf{2 7} \cdot \mathbf{2 7} \cdot \mathbf{2} \mathbf{7}_{H}$ with the third family coupling strength. We included the color factor and contributions of $\mathbf{1 0} \subset \mathbf{2 7}$ of the loop particles. For a simple numerical comparison, we assumed $C_{2}\left(R_{i}\right)=C_{2}(r)$ in Eq. (13). We set $\delta=2$, i.e. $D=6$ and $X_{2}=\pi$. 
For Eq. (12), we consider first $\Lambda \simeq M_{G U T}$, and then consider for a general $\Lambda$. For example, consider one $\mathbf{2 7}$ of $E_{6}$ in the bulk. Then, for Eqs. (12) and (13) we obtain at $\Lambda \simeq M_{G U T}$

$$
28[\pi-1] Y^{2} \simeq 29.4
$$

and

$$
-12 C_{2}(\mathbf{2 7}) G_{2}^{2}=-163
$$

respectively, using $Y \simeq 0.7$. In Eq. (15), we used $G_{\delta}$ evaluated at $\Lambda=M_{G U T}$. Thus, near the scale $M_{G U T}$ Eq. (13) is the dominant term and the Yukawa coupling decreases as the scale increases. For $Y^{2}(\Lambda)$ evaluation, we need

$$
\left(\frac{g\left(M_{G U T}\right)}{g(\Lambda)}\right)^{2}=1+\frac{C_{2}(G) X_{\delta} g^{2}\left(M_{G U T}\right)}{4 \pi^{2} \delta}\left\{\left(\frac{\Lambda}{M_{G U T}}\right)^{\delta}-1\right\} .
$$

Then, $Y^{2}(\Lambda)$ is

$$
\begin{aligned}
Y^{2}(\Lambda) & =\left(\frac{g(\Lambda)}{g\left(M_{G U T}\right)}\right)^{2 \eta_{Y}^{i j k}} Y^{2}\left(M_{G U T}\right) \\
& =\left[1+\frac{C_{2}(G) X_{\delta} g^{2}\left(M_{G U T}\right)}{4 \pi^{2} \delta}\left\{\left(\frac{M_{P}}{M_{G U T}}\right)^{\delta}-1\right\}\right]^{-\eta_{Y}^{i j k}} Y^{2}\left(M_{G U T}\right) \\
& \simeq\left[1+\frac{6}{25}\left\{\left(\frac{\Lambda}{M_{G U T}}\right)^{2}-1\right\}\right]^{-13 / 6}(0.7)^{2}
\end{aligned}
$$

where $\eta_{Y}^{i j k}=\left(C_{2}\left(R_{i}\right)+C_{2}\left(R_{j}\right)+C_{2}\left(R_{k}\right)\right) / C_{2}\left(G_{a}\right)$. For the last equality, we used $C_{2}\left(E_{6}\right)=12$ and $Y\left(M_{G U T}\right)=0.7$. Thus, Eq. (12) becomes

$$
28\left[X_{\delta}\left(\frac{\Lambda}{M_{G U T}}\right)^{2}-1\right]\left[1+\frac{6}{25}\left\{\left(\frac{\Lambda}{M_{G U T}}\right)^{2}-1\right\}\right]^{-13 / 6}(0.7)^{2}
$$

which can be compared to the magnitude given in (13) which is saturated to

$$
-96 \pi^{2} \frac{C_{2}(\mathbf{2 7})}{C_{2}(\mathbf{7 8})}=-684
$$

The maximum value of $(18)$ is about $54 \simeq 2 M_{G U T}$. Note the value of Eq. (13) is -384 at $\Lambda \simeq 2 M_{G U T}$. Therefore, Eq. (12) can never exceed the magnitude of (13). Therefore, 
Eq. (13) continues to dominate Eq. (12). Hence, we can approximate the Yukawa coupling running given by the third term of Eq. (10), leading to

$$
Y^{i j k}\left(M_{G U T}\right)=\left(\frac{g\left(M_{G U T}\right)}{g\left(M_{s}\right)}\right)^{\eta_{Y}^{i j k}} Y^{i j k}\left(M_{s}\right)
$$

This is a multiplicative result and the needed inter-family mixings are not suppressed.

For the other soft terms $\left(m^{2}, A\right.$ and $B$ terms) we have checked the contributions of $Y^{2}$ to the evolution equations are also negligible, and bulk matter can be allowed toward a successful KT mechanism.

\section{THE ANOMALY}

In the previous section, the softening of the SUSY flavor problem has been obtained by a large number of $M_{a}\left(M_{G U T}\right) / M_{a}\left(M_{s}\right)$ which depends on $\left[g\left(M_{G U T}\right) / g\left(M_{s}\right)\right]^{2}$ which in turn depends on $\sim\left(M_{s} / M_{G U T}\right)^{\delta}$. Given the small number of order $10-100$ for $M_{s} / M_{G U T}$, a larger $\delta$ can remove the unwanted flavor violating pieces more effectively. We argure, in accord with Kubo and Terao, that $\delta=1$ is not sufficient. ${ }^{5}$ Thus, we consider $\delta=2$, i.e. $6 \mathrm{D}$ SUSY field theories.

Then, we search for models satisfying two conditions: (i) no gauge anomaly, and (ii) asymptotic freedom in the bulk.

One should consider also the gravitational anomaly [15], but it is easy to remove it by adding gauge singlet fermions. Thus, we will not use the vanishing gravitational anomaly as an absolute condition.

Note that there exist square anomalies in $6 \mathrm{D}$ [14]. We are interested in the $A, D, E$ series. The asymptotic freedom condition is calculated from the fields in the bulk. The

\footnotetext{
${ }^{5}$ If $\delta=1$, some flavor changing problems can be evaded but the SUSY CP problem is difficult to understand [13] with the extra dimensional scenario alone. However, if $1 / R \ll M_{G U T}$ is assumed, $\delta=1$ can be admissible.
} 
gauge multiplet splits into an $N=1$ gauge multiplet plus a chiral multiplet in $4 \mathrm{D}$, and a hyper multiplet splits into two chiral multiplets with opposite quantum numbers in 4D. Thus, we require

$$
-2 C_{2}(G)+\sum_{i} 2 \ell\left(R_{i}\right)<0
$$

where $\ell\left(R_{i}\right)$ is the index of the representation $R_{i}$, and the sum is for the bulk hyper multiplet representations.

\section{A. $S U(N)$ and $S O(2 n)$}

The groups $S U(N)$ and $S O(2 n)$ have the following anomalies for the same chirality fermions,

$$
\begin{aligned}
S U(N): & 2 N \text { (for adjoint), } \\
& \left.\frac{(N-4) ![N(N+1)-6 j(N-j)]}{(j-1) !(N-1-j) !} \text { (for } j \text { antisymmetric indices }[j]\right) \\
& 1 \text { (for fundamental) } \\
S O(2 n): & 4(n-4) \text { (for adjoint) } \\
& -2^{n-4} \text { (for spinor) } \\
& 2 \text { (for fundamental) }
\end{aligned}
$$

Note, however, that the vector multiplet and hyper multiplets have the opposite chiralities to be consistent with supersymmetry. Most models satisfying the anomaly free condition do not satisfy the asymtotic freedom condition. Note that among the models presented in Ref. [16] the $S U(5)$ (ten $\mathbf{5}$ in the bulk) is not allowed but $S O(10)$ (three 10's and one $\overline{\mathbf{1 6}}$ ) is allowed.

We have not found any $6 \mathrm{D} S U(N)$ model satisfying the two conditions. In $S O(10)$, there

are two models: one model with two 10's and the other with three 10's and one $\mathbf{1 6}$ (or $\overline{\mathbf{1 6}}$ ). For $S O(2 n)$ with $n \geq 6$, there always exists one model: $2(n-4)$ fundamental representations. 


\section{B. Exceptional groups}

But more interesting groups are the exceptional groups. It is known that the exceptional groups are anomaly free in 6D [14]. In this sense, the exceptional groups in the $E$-series can be claimed to be the grand unification groups in $6 \mathrm{D}$, as the orthogonal groups in the $D$-series are considered as the grand unfication groups in $4 \mathrm{D}$. In fact an anomaly-free $E_{7}$ 6D model was obtained by a $Z_{2}$ orbifold compactification of the heterotic string [3]. Its $E_{7}$

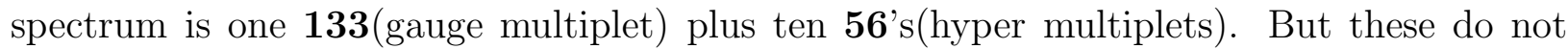
satisfy the asymptotic freedom condition in the bulk.

Here, we consider any $6 \mathrm{D} E_{6}, E_{7}, E_{8}$ field theoretic models in the bulk, hoping that they can be obtained from some compactification of string models. For these, we obtain constraints for the number of fundamental representations in the bulk matter

$$
\begin{aligned}
& E_{6}: n_{27} \leq 3 \\
& E_{7}: n_{56} \leq 2 \\
& E_{8}: \text { no hyper multiplet in the bulk }
\end{aligned}
$$

where we used $C_{2}\left(E_{i}\right)=(12,18,30)$ for $(i=6,7,8)$, respectively, and $\ell\left(\mathbf{2 7}_{E_{6}}\right)=3$ and $\ell\left(\mathbf{5 6}_{E_{7}}\right)=6$. Thus, in principle it is possible to put all chiral matter representations in the bulk.

It will be of utmost importance to search for 6D SUSY models with the above property through the string compactifications. They can be considered as the string solutions of the SUSY flavor problem.

\section{CONCLUSION}

We have considered $6 \mathrm{D}$ groups with the asymptotic freedom in the bulk, toward softening

of the SUSY flavor problem. We extended the KT mechanism to include the possibility of hyper multiplets in the bulk. The two conditions on the anomaly freedom and asymptotic 
freedom exclude most 6D GUT models, except two $S O(10)$ models, $S O(2 n)$ models(one each for each $n \geq 6$ ), and several exceptional group models. The suppression of the flavor changing neutral current is obtained because of the existence of a small number (1) due to a large internal 6D volume compared to the Planckian volume(or the string scale volume).

\section{ACKNOWLEDGMENTS}

We thank Hyun Min Lee and Hans Peter Nilles for useful discussions. One of us(JEK) thanks Humboldt Foundation for the award. This work is supported in part by the BK21

program of Ministry of Education, KOSEF Sundo Grant, and Korea Research Foundation Grant No. KRF-PBRG-2002-070-C00022. 


\section{REFERENCES}

[1] See, for example, M. Dine and Ann Nelson, Phys. Rev. D48 (1993) 1277 [hepph/9303230].

[2] Y. Kawamura, Prog. Theor. Phys. 105 (2001) 999 [hep-ph/0012125].

[3] K.-S. Choi and J. E. Kim, Phys. Lett. B552 (2003) 81 [hep-th/0206099].

[4] V. Kaplunovsky, Phys. Rev. Lett. 55 (1985) 1036.

[5] P. Horava and E. Witten, Nucl. Phys. B460 (1995) 506 [hep-th/9510209].

[6] T. Friedmann and E. Witten, hep-th/0211269.

[7] T. R. Taylor and G. Veneziano, Phys. Lett. B212 (1988) 47; K. Dienes, E. Dudas and T. Gherghetta, Phys. Lett. B436 (1998) 55 [hep-ph/9803466].

[8] J. Kubo and H. Terao, Phys. Rev. D66 (2002) 116003 [hep-ph/0205247].

[9] H. B. Kim and J. E. Kim, Phys. Lett. B300 (1993) 343 [hep-ph/9212311].

[10] H. D. Kim, J. E. Kim and H. M. Lee, Euro. Phys. J. C24 (2002) 159 [hep-ph/0112094].

[11] For tables for 4D orbifold models, see Y. Katsuki, Y. Kawamura, T. Kobayashi, N. Ohtsubo, Y. Ono, and K. Tanioka, Kanazawa Univ. preprint DPKU-8904 (1989). For tables for 6D orbifold models, see L. Ibanez and A. M. Uranga, hep-th/9707075.

[12] J. Kubo and M. Nunami, Phys. Rev. D63 (2001) 116011 [hep-ph/0011147].

[13] F. Gabbiani, E. Gabrielli, A. Masiero, and L. Silvestrini, Nucl. Phys. B477 (1996) 321 [hep-ph/9604387].

[14] P. Frampton and T. Kephart, Phys. Rev. D28 (1983) 1010; S. Okubo and Y. Tosa, Phys. Rev. D36 (1987) 2484; A. Hebecker and J. March-Russell, Nucl. Phys. B625 (2002) 128 [hep-ph/0107039].

[15] L. Alvarez-Gaume and E. Witten, Nucl. Phys. B234 (1984) 269. 
[16] T. Watari and T. Yanagida, Phys. Lett. B519 (2001) 164 [hep-ph/0108152]; T. Asaka, W. Buchmüller and L. Covi, Phys. Lett. B540 (2002) 295 [hep-ph/0204358]. 